\title{
Review of: "Comparison of methods to experimentally induce opacification and elasticity change in ex vivo porcine lenses"
}

\author{
Douglas Borchman ${ }^{1}$
}

1 University of Louisville

Potential competing interests: The author(s) declared that no potential competing interests exist.

The major contribution of this study is that a porcine lens culture system was established and the opacity and elasticity were measured after cataracts were induced with $\mathrm{NaCL}$, formalin and microwave heating. The manuscript was well written and the science and conclusions were supported by the data. I have a few major concerns:

1. The elasticity measurements were unique, however, similar studies have been performed and the advantages and disadvantages of the current study were not discussed or related to previous studies (See J. Nutrit. Therap. 2018;7:31-39. Curr Eye Res. June 6:1-6, 2018, Exp. Eye Res. 170:67-75, 2018). A multitude of organ culture studies have been done and have been reviewed but not mentioned. See the introduction in Exp. Eye Res. 170:67-75, 2018. How does the current study add to the previous studies? 2. My enthusiasm for the study was low as the rational for the study is not strong. There are almost no drug companies or laboratories interested in testing drugs to ameliorate cataracts since surgery is cheap, safe and quick whereas drugs may be expensive and have systemic affects.

3. Although animal lenses are usually cheaper, more readily obtained than human lenses, human lenses differ significantly from animal lenses so the use of human lens models is significantly advantageous over animal models [Truscott, 2005; Borchman et al., 2004]. For instance human lenses differ from animal lenses in regards to UV filters, oxidation with age, protein content, crystalline content, compaction with age, synthesis of ascorbate, antioxidant enzymes [Truscott, 2005] and phospholipid composition and membrane structure [Borchman et al., 2004].

Truscott RJW. Age-related nuclear cataract-oxidation is the key. Experimental Eye Research 80 (2005) 709-725.

Borchman D, Yappert MC, Afzal M. Lens lipids and maximum lifespan. Exp Eye Res. 2004 Dec;79(6):761-8. 4. Measuring light scattering and absorbance may be more quantitative than subjectively grading lens opacity visually as the cortex could be cloudy but the nuclear region clear obfuscating the overall opacity grade.

5. A minor point, RPMI, DMEM should be defined the $1^{\text {st }}$ time they appear.

6. Lens hardening may also be a result of lens membranes becoming stiffer (J. Lipid Res. 2021; 62:100039, J. Lipid Res. 51:2473-88, 2010. 
